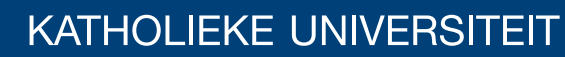 \\ LEUVEN
}

\section{Faculty of Business and Economics}

S-estimation for penalized regression splines

K. Tharmaratnam, G. Claeskens, C. Croux and M. Salibián-Barrera

DEPARTMENT OF DECISION SCIENCES AND INFORMATION MANAGEMENT (KBI) 


\title{
S-Estimation for Penalized Regression Splines
}

\author{
K.Tharmaratnam, G. Claeskens and C. Croux \\ OR \& Business Statistics and Leuven Statistics Research Center \\ Katholieke Universiteit Leuven, Belgium
}

M. Salibián-Barrera

Department of Statistics, University of British Columbia, Canada

October 2008

\begin{abstract}
This paper is about S-estimation for penalized regression splines. Penalized regression splines are one of the currently most used methods for smoothing noisy data. The estimation method used for fitting such a penalized regression spline model is mostly based on least squares methods, which are known to be sensitive to outlying observations. In real world applications, outliers are quite commonly observed. There are several robust estimation methods taking outlying observations into account. We define and study S-estimators for penalized regression spline models. Hereby we replace the least squares estimation method for penalized regression splines by a suitable S-estimation method. By keeping the modeling by means of splines and by keeping the penalty term, though using S-estimators instead of least squares estimators, we arrive at an estimation method that is both robust and flexible enough to capture non-linear trends in the data. Simulated data and a real data example are used to illustrate the effectiveness of the procedure.
\end{abstract}

Keywords: M-estimator, Penalized least squares method, Penalized regression spline, S-estimator, Smoothing parameter 


\section{Introduction}

Penalized regression spline models have found a lot of applications in the last 10-15 years. Their ease of fitting and flexible choice of knots and smoothing parameter has made them a popular nonparametric smoothing method. The use of a combination of regression splines, which have a substantially smaller number of knots than the sample size, and the use of a penalty, dates back to at least O'Sullivan (1986) who used a cubic B-spline basis for estimation in inverse problems. Hybrid splines, which approximate the smoothing splines (the latter which have knots equal to the data points and a penalty for complexity) have been studied by Kelly and Rice (1990) and Besse et al. (1997). Eilers and Marx (1996) proposed the use of a difference penalty on the spline coefficients. For more explanation and examples on the class of penalized regression spline models, we refer to Ruppert et al. (2003). Theoretical aspects of penalized spline regression fitting are only recently starting to develop. We refer to Hall and Opsomer (2005) for a white noise representation of the model, Claeskens et al. (2007) for relating theoretical properties of penalized regression splines to those of regression splines (without a penalty) and smoothing splines, and Kauermann et al. (2007) for results in generalized linear models.

The estimation method used for fitting such penalized regression spline models minimizes the sum of squared residuals subject to a bound on the norm of the spline regression coefficients. Alternatively, one can work with the equivalent penalized minimization problem, that has a closed-form expression for its solution. It is easy to see that this approach may be highly sensitive to the presence of a small proportion of atypical observations. One way to obtain a fit that is more resistant to the effect of atypical observations in the data is to replace the squared residuals by a slowly increasing loss function, as it is done for M-regression estimators (Huber, 1964). Early proposals dealing with M-type robust smoothing go back to Huber (1979) and Cox (1983) for the particular case of cubic regression splines. Other papers on the topic include Härdle and Gasser (1984), Silverman (1985) and Hall and Jones (1990). More recently Oh et al. (2004, 2007) used the "pseudo values" introduced in Cox (1983) to derive iterative algorithms for M-type cubic splines, while Lee and Oh (2007) applied this approach to M-penalized regression splines.

As already noted by Huber (1979) and Cox (1983), a serious difficulty with replacing the squared residuals by a slower-increasing loss function to obtain M-type smoothers is 
that one needs to either know or robustly estimate the residual scale. In principle, one can use simultaneous estimation of the regression and scale parameters (Huber's Proposal II (Huber, 1964)), as in Lee and Oh (2007). Unfortunately, our numerical experiments show that, as in the simple location/scale and linear regression models, simultaneous estimation of the regression coefficients and the residual scale may not have good robustness properties. In particular, the procedure may be seriously affected by a relatively small proportion of outliers.

The main purpose of this paper is to propose robust penalized regression splines that are able to resist the potentially damaging effect of outliers in the sample, and that do not require the separate estimation of the residual scale. To achieve these goals we propose to compute penalized S-regression estimators. In the unpenalized case, these estimators are consistent, asymptotically normal, and have high-breakdown point regardless of the dimension of the vector of regression coefficients (Rousseeuw and Yohai, 1984).

First we show that the solution to the penalized S-regression problem can be written as the solution of a weighted penalized least squares problem. This representation naturally leads to an iterative algorithm to compute these estimators. We also study how to robustly select the penalty parameter when there may be outliers in the data. This is a difficult problem that was studied for M-cubic splines by Cantoni and Ronchetti (2001). We propose a robust penalty parameter selection criteria based on generalized cross-validation that also borrows from the weighted penalized least squares representation of the penalized S-regression estimator. Extensive simulation studies show that our algorithm works well in practice and that the resulting regression function estimator is robust to the presence of outliers in the data. Furthermore, these estimators compare favorably to the M-penalized splines of Lee and Oh (2007).

The rest of this paper is organized as follows. Section 2 introduces penalized Sestimators and an algorithm to compute them, while Section 3 reports the results of a simulation study that compared the performance of classical least-squares, M- and Spenalized regression estimators. A real data set is analyzed in Section 4 and concluding remarks are included in Section 5. 


\section{Penalized S-regression splines}

Consider the regression model

$$
Y=m(x)+\varepsilon,
$$

where $m:[a, b] \rightarrow \mathbb{R}$ is an unknown but smooth regression function and the random error $\varepsilon$ is independent from the explanatory variable $x \in \mathbb{R}$, and has mean zero and constant variance $\sigma^{2}$. We are interested in estimating the function $m(x)$ based on a random sample $\left(Y_{i}, x_{i}\right), i=1, \ldots, n$.

A widely used estimation method for $m(x)$ is to assume that

$$
m(x)=\sum_{j=1}^{L} \beta_{j} f_{j}(x)
$$

for some basis $f_{1}(x), \ldots, f_{L}(x)$ and coefficients $\beta_{j} \in \mathbb{R}$. To fix ideas, we focus our presentation on truncated polynomial bases, but other choices can be used as well. More specifically, we take $K$ inner knots $a<\kappa_{1}<\cdots<\kappa_{K}<b$ and define

$$
m(x ; \boldsymbol{\beta})=\beta_{0}+\beta_{1} x+\cdots+\beta_{p} x^{p}+\sum_{j=1}^{K} \beta_{p+j}\left(x-\kappa_{j}\right)_{+}^{p},
$$

where $a_{+}=\max (a, 0)$ and $\boldsymbol{\beta}=\left(\beta_{0}, \beta_{1}, \ldots, \beta_{p+K}\right)^{t}$. Given a sample $\left(Y_{1}, x_{1}\right), \ldots,\left(Y_{n}, x_{n}\right)$ this approach transforms the estimation of $m(\cdot)$ into a least squares problem, where we find the member of the class $m(x ; \boldsymbol{\beta})$ that minimizes the sum of squared residuals. To avoid overfitting, we solve the problem subject to a bound on the size of the spline coefficients:

$$
\min _{\boldsymbol{\beta} \in \mathbb{R}^{p+K+1}} \sum_{i=1}^{n}\left(Y_{i}-m\left(x_{i} ; \boldsymbol{\beta}\right)\right)^{2} \quad \text { subject to } \sum_{j=1}^{K} \beta_{p+j}^{2} \leq C,
$$

for some $C>0$ as in Ruppert et al. (2003). If we let $\boldsymbol{F}(x)=\left(1, x, \ldots, x^{p},(x-\right.$ $\left.\left.\kappa_{1}\right)_{+}^{p}, \ldots,\left(x-\kappa_{K}\right)_{+}^{p}\right)^{t} \in \mathbb{R}^{p+K+1}$, it is easy to see that the penalized least squares regression spline estimator $\hat{\boldsymbol{\beta}}$ is the minimizer of

$$
\sum_{i=1}^{n}\left(Y_{i}-\boldsymbol{F}\left(x_{i}\right)^{t} \boldsymbol{\beta}\right)^{2}+\lambda \sum_{j=1}^{K} \beta_{p+j}^{2}
$$

for some penalty parameter $\lambda>0$. 
Denoting the spline design matrix $\boldsymbol{F}=\left\{\boldsymbol{F}\left(x_{1}\right)^{t}, \ldots, \boldsymbol{F}\left(x_{n}\right)^{t}\right\}^{t}$, the vector of responses $\boldsymbol{Y}=\left(Y_{1}, \ldots, Y_{n}\right)^{t}$ and $\boldsymbol{D}_{p}=\operatorname{diag}\left(0_{p+1}, 1_{K}\right)$ the matrix indicating that only the spline coefficients are to be penalized, the resulting estimator $\widehat{\boldsymbol{\beta}}$ is given by the ridge regression formula

$$
\widehat{\boldsymbol{\beta}}=\left(\boldsymbol{F}^{t} \boldsymbol{F}+\lambda \boldsymbol{D}\right)^{-1} \boldsymbol{F}^{t} \boldsymbol{Y},
$$

and the corresponding estimated vector $\widehat{\boldsymbol{m}}=\left(\hat{m}\left(x_{1}\right), \ldots, \hat{m}\left(x_{n}\right)\right)^{t}$ :

$$
\widehat{\boldsymbol{m}}=\boldsymbol{F} \widehat{\boldsymbol{\beta}}=\boldsymbol{F}\left(\boldsymbol{F}^{t} \boldsymbol{F}+\lambda \boldsymbol{D}\right)^{-1} \boldsymbol{F}^{t} \boldsymbol{Y}
$$

\subsection{Penalized S-estimation}

It is easy to see that, as in unpenalized linear regression, the estimator defined by the minimum of (3) may be seriously affected by a small proportion of atypical observations. These "outliers" may be errors in the data, or, more interestingly, data points that follow a different model or random process. In what follows we will be concerned with estimating the regression function $m(x)$ in (1) that applies to the majority of the data.

A straightforward approach to obtain penalized regression estimators that are more resistant to outliers than those defined by (3) is to replace the squared residual loss function by a slowly increasing function $\rho$ :

$$
\sum_{i=1}^{n} \rho\left(Y_{i}-\boldsymbol{F}\left(x_{i}\right)^{t} \boldsymbol{\beta}\right)+\lambda \sum_{j=1}^{K} \beta_{p+j}^{2},
$$

where $\rho$ is even, non-decreasing in $[0, \infty)$ and $\rho(0)=0$. Intuitively, the function $\rho(t)$ should increase at a slower rate than $t^{2}$, particularly for large residuals. A common choice for $\rho$ in (6) is given by Huber's family

$$
\rho_{c}(t)= \begin{cases}t^{2} & \text { if }|t| \leq c \\ 2 c|t|-c^{2} & \text { if }|t|>c\end{cases}
$$

where $c>0$ is a tuning constant. The parameter $c$ can be thought of as a threshold such that observations with residuals larger than $c$ have a reduced effect on the estimating equation (6). Note that as $c$ increases, the minimum of (6) approaches that of (3). In other words, the estimator downweights the influence of observations with large residual (i.e. larger than $c$ ). 
To apply this method in practice, we need to select a value of $c$ depending on $\sigma$, the standard deviation of the errors $\varepsilon$ in (1). This can be easily done if a robust scale estimator $\hat{\sigma}_{n}$ of $\sigma$ is available. In this case we can compute our estimator using the standardized residuals:

$$
\hat{\boldsymbol{\beta}}_{n}=\underset{\boldsymbol{\beta}}{\operatorname{argmin}} \sum_{i=1}^{n} \rho_{c}\left(\frac{Y_{i}-\boldsymbol{F}\left(x_{i}\right)^{t} \boldsymbol{\beta}}{\hat{\sigma}_{n}}\right)+\lambda \sum_{j=1}^{K} \beta_{p+j}^{2} .
$$

Given a set of residuals $r_{i}=Y_{i}-\boldsymbol{F}\left(x_{i}\right)^{t} \hat{\boldsymbol{\beta}}_{n}, i=1, \ldots, n$, corresponding to an estimator $\hat{\boldsymbol{\beta}}_{n}$, a robust M-scale estimator $\hat{\sigma}_{n}$ (Huber, 1964) satisfies

$$
\frac{1}{n} \sum_{i=1}^{n} \rho\left(\frac{r_{i}}{\hat{\sigma}_{n}}\right)=b
$$

where, $\rho: \mathbb{R} \rightarrow[0, \infty)$ is bounded and even and, to obtain consistency when the errors are normal, the constant $b$ satisfies $b=E_{\Phi}[\rho(Z)]$, with $\Phi$ the standard normal distribution. Note that if $\rho(t)=t^{2}$ and $b=1$ then $\hat{\sigma}_{n}=s_{n}$ the residual standard deviation.

Huber (1964) proposed to simultaneously solve the "regression" and "scale" equations, (8) and (9), respectively. In our context this is equivalent to finding the solutions $\hat{\boldsymbol{\beta}}_{n}$ and $\hat{\sigma}_{n}$ to the following non-linear system of equations (see also Lee and Oh, 2007):

$$
\begin{gathered}
\left.\frac{\partial}{\partial \boldsymbol{\beta}}\left(\sum_{i=1}^{n} \rho_{c}\left(\frac{Y_{i}-\boldsymbol{F}\left(x_{i}\right)^{t} \boldsymbol{\beta}}{\hat{\sigma}_{n}}\right)+\lambda \sum_{j=1}^{K} \beta_{p+j}^{2} \cdot\right)\right|_{\boldsymbol{\beta}=\hat{\boldsymbol{\beta}}_{n}}=\mathbf{0} \\
\frac{1}{n} \sum_{i=1}^{n} \rho\left(\frac{Y_{i}-\boldsymbol{F}\left(x_{i}\right)^{t} \hat{\boldsymbol{\beta}}_{n}}{\hat{\sigma}_{n}}\right)=b .
\end{gathered}
$$

Finding $\hat{\boldsymbol{\beta}}_{n}$ and $\hat{\sigma}_{n}$ generally requires using an iterative algorithm. This scheme is known in the robustness literature as Huber's Proposal II. Unfortunately, the robustness properties of the solution to this problem are not completely satisfactory. In particular, the resulting estimators may not be resistant to outliers, i.e. they have low breakdown point (see Donoho and Huber (1983) for a definition of breakdown point). This was shown by Maronna and Yohai (1991) for simultaneous general M-estimators of regression and scale.

S-estimators for linear regression were introduced by Rousseeuw and Yohai (1984). They can be tuned to have a high breakdown point and do not require an auxiliary residual scale estimator. The basic idea is to note that the least squares estimator is the 
vector of regression coefficients that produces residuals with minimal sample standard deviation.

A robust alternative is then obtained by finding the vector of regression coefficients $\boldsymbol{\beta}$ that produces residuals that minimize a robust scale estimator of the residuals, instead of the standard deviation. In other words, the S-estimators are defined by

$$
\hat{\boldsymbol{\beta}}_{n}=\underset{\boldsymbol{\beta}}{\operatorname{argmin}} \hat{\sigma}_{n}(\boldsymbol{\beta})
$$

where $\hat{\sigma}_{n}(\boldsymbol{\beta})$ is an M-scale that solves (9). It is easy to see that $\hat{\sigma}_{n}=\hat{\sigma}_{n}(\hat{\boldsymbol{\beta}})$ is also a consistent estimator of the scale $\sigma$ of the errors. For linear regression models, Rousseeuw and Yohai (1984) and Davies (1990) showed that S-estimators are consistent and asymptotically normal when the distribution of the errors is symmetric.

Note that there is no explicit formula to compute $\hat{\sigma}(\boldsymbol{\beta})$ for each $\boldsymbol{\beta}$. Furthermore if $\rho$ is bounded, then the function $\sigma(\boldsymbol{\beta})$ is non-convex, and may have several local minima. Solving (10) is a difficult numerical problem that involves finding the minimum of an implicitly defined non-convex function in several variables. A recently proposed algorithm for unpenalized S-regression estimators can be found in Salibian-Barrera and Yohai (2006).

One way to obtain robust penalized spline estimators is to replace the mean squared residuals in (3) by a robust estimator of the scale of residuals. In this paper we consider using the S-scale, which can naturally be seen as a penalized S-splines estimator.

More specifically, we define $\hat{\boldsymbol{\beta}}_{S}$ as

$$
\hat{\boldsymbol{\beta}}_{S}=\underset{\boldsymbol{\beta}}{\operatorname{argmin}}\left[n \hat{\sigma}_{n}^{2}(\boldsymbol{\beta})+\lambda \boldsymbol{\beta}^{t} \boldsymbol{D} \boldsymbol{\beta}\right]
$$

where, for each $\boldsymbol{\beta}, \hat{\sigma}_{n}(\boldsymbol{\beta})$ satisfies

$$
\frac{1}{n} \sum_{i=1}^{n} \rho\left(\frac{Y_{i}-\boldsymbol{F}\left(x_{i}\right)^{t} \boldsymbol{\beta}}{\hat{\sigma}_{n}(\boldsymbol{\beta})}\right)=b
$$

the constant $b=E_{\Phi}[\rho(Z)]$, and $\Phi$ is the standard normal distribution (Maronna and Yohai, 2006).

A commonly used family of loss functions $\rho$ is given by Tukey's bi-square family (Beaton and Tukey, 1974)

$$
\rho_{d}(u)= \begin{cases}3(u / d)^{2}-3(u / d)^{4}+(u / d)^{6} & \text { if }|u| \leq d \\ 1 & \text { if }|u|>d\end{cases}
$$


The choice $d=1.5476$ yields $b=E_{\Phi}[\rho(Z)]=0.50$. The associated unpenalized Sregression estimator has maximal asymptotic breakdown point $50 \%$ (Rousseeuw and Yohai, 1984).

The next result shows that the critical points of the objective function in (11) can be written as the solution of a weighted penalized splines problem. This expression suggests an iterative procedure to compute the penalized S-estimators.

Result 1 The penalized S-estimator for the regression spline model (1) can be written as $\widehat{\boldsymbol{m}}_{S}=\boldsymbol{F} \widehat{\boldsymbol{\beta}}_{S}$ where

$$
\widehat{\boldsymbol{\beta}}_{S}=\left\{\boldsymbol{F}^{t} \boldsymbol{W}\left(\widehat{\boldsymbol{\beta}}_{S}\right) \boldsymbol{F}+\frac{\lambda}{\tau\left(\widehat{\boldsymbol{\beta}}_{S}\right)} \boldsymbol{D}\right\}^{-1} \boldsymbol{F}^{t} \boldsymbol{W}\left(\widehat{\boldsymbol{\beta}}_{S}\right) \boldsymbol{Y},
$$

where $\boldsymbol{W}(\boldsymbol{\beta})=\operatorname{diag}\left(W_{i}(\boldsymbol{\beta})\right) \in \mathbb{R}^{n \times n}$ with $W_{i}(\boldsymbol{\beta})=\rho^{\prime}\left(\tilde{r}_{i}(\boldsymbol{\beta})\right) / \tilde{r}_{i}(\boldsymbol{\beta}), \tilde{r}_{i}(\boldsymbol{\beta})=\left(Y_{i}-\right.$ $\left.\boldsymbol{F}\left(x_{i}\right)^{t} \boldsymbol{\beta}\right) / \hat{\sigma}_{n}(\boldsymbol{\beta})$, and $\tau(\boldsymbol{\beta})=n \hat{\sigma}_{n}^{2}(\boldsymbol{\beta}) /\left[(\boldsymbol{Y}-\boldsymbol{F} \boldsymbol{\beta})^{t} \boldsymbol{W}(\boldsymbol{\beta})(\boldsymbol{Y}-\boldsymbol{F} \boldsymbol{\beta})\right]$.

Proof of Result 1: Taking the derivative with respect to $\boldsymbol{\beta}$ for $\hat{\sigma}_{n}(\boldsymbol{\beta}) \neq 0$ of the M-scale function in (12), we obtain

$$
\sum_{i=1}^{n} \rho^{\prime}\left(\frac{r_{i}(\boldsymbol{\beta})}{\hat{\sigma}_{n}(\boldsymbol{\beta})}\right)\left(\frac{-\boldsymbol{F}\left(x_{i}\right) \hat{\sigma}_{n}(\boldsymbol{\beta})-r_{i}(\boldsymbol{\beta}) \nabla \hat{\sigma}_{n}(\boldsymbol{\beta})}{\hat{\sigma}_{n}^{2}(\boldsymbol{\beta})}\right)=\mathbf{0},
$$

where $\nabla \hat{\sigma}_{n}(\boldsymbol{\beta})=\partial \hat{\sigma}_{n}(\boldsymbol{\beta}) / \partial \boldsymbol{\beta}$. It follows that

$$
\begin{aligned}
\nabla \hat{\sigma}_{n}(\boldsymbol{\beta}) & =-\sum_{i=1}^{n} \rho^{\prime}\left(\frac{r_{i}(\boldsymbol{\beta})}{\hat{\sigma}_{n}(\boldsymbol{\beta})}\right) \boldsymbol{F}\left(x_{i}\right) /\left[\sum_{i=1}^{n} \rho^{\prime}\left(\frac{r_{i}(\boldsymbol{\beta})}{\hat{\sigma}_{n}(\boldsymbol{\beta})}\right)\left(\frac{r_{i}(\boldsymbol{\beta})}{\hat{\sigma}_{n}(\boldsymbol{\beta})}\right)\right] \\
& =\left[-\hat{\sigma}_{n}(\boldsymbol{\beta}) \boldsymbol{F}^{t} \boldsymbol{W}(\boldsymbol{\beta}) \boldsymbol{r}(\boldsymbol{\beta})\right] /\left[\boldsymbol{r}(\boldsymbol{\beta})^{t} \boldsymbol{W}(\boldsymbol{\beta}) \boldsymbol{r}(\boldsymbol{\beta})\right]
\end{aligned}
$$

where $\boldsymbol{r}(\boldsymbol{\beta})=\left(\boldsymbol{Y}-\boldsymbol{F}^{t} \boldsymbol{\beta}\right)$. At the minimum of (11) $\hat{\boldsymbol{\beta}}_{S}$ we have

$$
2 n \hat{\sigma}_{n}\left(\hat{\boldsymbol{\beta}}_{S}\right) \nabla \hat{\sigma}_{n}\left(\hat{\boldsymbol{\beta}}_{S}\right)+2 \lambda \boldsymbol{D} \hat{\boldsymbol{\beta}}_{S}=\mathbf{0},
$$

from which follows, using (15) that

$$
-\tau\left(\hat{\boldsymbol{\beta}}_{S}\right) \boldsymbol{F}^{t} \boldsymbol{W}\left(\hat{\boldsymbol{\beta}}_{S}\right) \boldsymbol{r}\left(\hat{\boldsymbol{\beta}}_{S}\right)+\lambda \boldsymbol{D} \hat{\boldsymbol{\beta}}_{S}=\mathbf{0},
$$

and thus equation (14) follows. 
Remark 1 Note that both the weights and the penalty parameter on the right-hand side of (14) depend on $\widehat{\boldsymbol{\beta}}_{S}$ on the left of that equation. Although not useful for direct calculation of $\widehat{\boldsymbol{\beta}}_{S}$, this representation naturally suggests iterations of the form

$$
\widehat{\boldsymbol{\beta}}_{S, k+1}=\left\{\boldsymbol{F}^{t} \boldsymbol{W}\left(\widehat{\boldsymbol{\beta}}_{S, k}\right) \boldsymbol{F}+\lambda \boldsymbol{D} \tau\left(\widehat{\boldsymbol{\beta}}_{S, k}\right)^{-1}\right\}^{-1} \boldsymbol{F}^{t} \boldsymbol{W}\left(\widehat{\boldsymbol{\beta}}_{S, k}\right) \boldsymbol{Y}, \quad k=0,1,2, \ldots,
$$

to find critical points of (11). The corresponding algorithm is presented in the next section.

Remark 2 When $\rho(t)=t^{2}$ the $M$-scale estimator $\hat{\sigma}_{n}$ reduces to the sample standard deviation. In this case we have $\boldsymbol{W}(\boldsymbol{\beta})=2 \boldsymbol{I}_{n}$, where $\boldsymbol{I}_{n}$ is the $n \times n$ identity matrix, and $\tau(\boldsymbol{\beta})=1 / 2$. Hence, as expected, (14) reduces to the usual penalized least squares formula (4).

\section{$2.2 \quad$ Algorithm}

Although (14) suggests easily implementable iterations to calculate a critical point of (11), care should be taken as the function $\hat{\sigma}_{n}: \mathbb{R}^{p} \rightarrow \mathbb{R}_{+}$in (12) is generally non-convex. In other words, the objective function in (11) may have several critical points that only correspond to local minima. As a result, the iterations derived from Result 1 above may converge to different critical points (some of them non-optimal) depending on the starting value. As it is done for S-estimators for linear regression models, we propose to start the iterations from many initial points, and select the best resulting point (in terms of value of the objective function) as our approximate solution to the minimization problem (11).

Our algorithm can be described in the following steps:

Step (1) Let $\tilde{\boldsymbol{\beta}}_{1}^{(0)}, \ldots, \tilde{\boldsymbol{\beta}}_{J}^{(0)}$, be initial candidates. For each $\tilde{\boldsymbol{\beta}}_{j}^{(0)}$ :

(a) Compute $\widehat{\sigma}_{n}\left(\widehat{\boldsymbol{\beta}}_{j}^{(0)}\right), \tau\left(\widehat{\boldsymbol{\beta}}_{j}^{(0)}\right)$, and $\boldsymbol{W}\left(\widehat{\boldsymbol{\beta}}_{j}^{(0)}\right)$.

(b) Set $k=0$. Iterate the following steps:

(i) Let $\widehat{\boldsymbol{\beta}}_{j}^{(k+1)}=\left\{\boldsymbol{F}^{t} \boldsymbol{W}\left(\widehat{\boldsymbol{\beta}}_{j}^{(k)}\right) \boldsymbol{F}+\lambda \boldsymbol{D} \tau^{-1}\left(\widehat{\boldsymbol{\beta}}_{j}^{(k)}\right)\right\}^{-1} \boldsymbol{F}^{t} \boldsymbol{W}\left(\widehat{\boldsymbol{\beta}}_{j}^{(k)}\right) \boldsymbol{Y}$.

(ii) If either $k=\operatorname{maxit}$ (maximum number of iterations) or $\left\|\widehat{\boldsymbol{\beta}}_{j}^{(k)}-\widehat{\boldsymbol{\beta}}_{j}^{(k+1)}\right\|<$ $\epsilon\left\|\widehat{\boldsymbol{\beta}}_{j}^{(k)}\right\|$ where $\epsilon>0$ is a fixed small constant(the tolerance level, then set $\widehat{\boldsymbol{\beta}}_{j}^{F}=\widehat{\boldsymbol{\beta}}_{j}^{(k)}$ and break. 
(iii) Else, compute $\widehat{\sigma}_{n}\left(\widehat{\boldsymbol{\beta}}_{j}^{(k+1)}\right), \tau\left(\widehat{\boldsymbol{\beta}}_{j}^{(k+1)}\right), \boldsymbol{W}\left(\widehat{\boldsymbol{\beta}}_{j}^{(k+1)}\right)$ and set $k \leftarrow k+1$.

Step (2) Calculate the objective function for each $\widehat{\boldsymbol{\beta}}_{j}^{F}, j=1,2, \ldots, J$, and select the one with the lowest value, i.e. let

$$
\widehat{\boldsymbol{\beta}}_{S}=\underset{1 \leq j \leq J}{\operatorname{argmin}}\left[n \widehat{\sigma}_{n}^{2}\left(\widehat{\boldsymbol{\beta}}_{j}^{F}\right)+\lambda \widehat{\boldsymbol{\beta}}_{j}^{F} \boldsymbol{D} \widehat{\boldsymbol{\beta}}_{j}^{F}\right]
$$

The $J$ initial candidates $\tilde{\boldsymbol{\beta}}_{j}^{(0)}$ in Step 1 can be chosen in a number of ways. Intuitively we want them to correspond to different regions of the optimization domain. In linear regression problems, these initial points are generally chosen based on the sample. For example, if there are $d$ covariates, $J$ random subsamples of size $d+1$ are selected from the data, and $\tilde{\boldsymbol{\beta}}_{j}^{(0)}$ is set to the least squares fit of the $j$-th subsample. A similar approach can be applied here, where, to avoid ill-conditioned subsamples caused by the sparsity of the design matrix based on the spline basis in (2), we take subsamples of larger size, e.g. floor $(n / 5)$. Note that this set of $J$ initial candidates can also be extended to include the M- and classical penalized regression splines estimators at very little additional computational cost.

We have coded the above algorithm in R (R Development Core Team, 2008), and made it publicly available at http://www.stat.ubc.ca/ matias/penalised. In our experience the above algorithm converges without problems in the vast majority of the cases. The algorithm with $\varepsilon=10^{-6}$ and maxit $=500$ converges generally in less than 60 iterations. For all of our simulation experiments, see section 3.2, we have never encountered a situation where the algorithm for penalized S-estimation diverged.

\subsection{Penalty parameter selection}

To avoid overfitting the data, the penalty parameter $\lambda$ in (11) is often chosen so as to minimize an estimator of the resulting mean squared prediction error. Such an estimator can be computed by leave-one-out cross-validation. More specifically, for each value of $\lambda$, let

$$
\mathrm{CV}(\lambda)=\frac{1}{n} \sum_{i=1}^{n}\left(y_{i}-\widehat{m}\left(x_{i}\right)^{(-i)}\right)^{2}
$$


where $\widehat{m}(x)^{(-i)}$ is the regression estimator obtained without using the pair of observations $\left(y_{i}, x_{i}\right)$. To evaluate $\operatorname{CV}(\lambda)$ above it is not necessary to re-compute the estimator $\widehat{m}(x) n$ times. It has been shown in Ruppert et al. (2003) that

$$
\mathrm{CV}(\lambda)=\frac{1}{n} \sum_{i=1}^{n} \frac{\left(y_{i}-\widehat{m}\left(x_{i}\right)\right)^{2}}{\left(1-\boldsymbol{H}(\lambda)_{i, i}\right)^{2}},
$$

where $\boldsymbol{H}(\lambda)_{i, i}$ denotes the $i$-th diagonal element of the "hat"-matrix

$$
\boldsymbol{H}(\lambda)=\boldsymbol{F}\left(\boldsymbol{F}^{t} \boldsymbol{F}+\lambda \boldsymbol{D}\right)^{-1} \boldsymbol{F}^{t}
$$

with $\boldsymbol{F}$ and $\boldsymbol{D}$ as in (4). Furthermore, if one replaces each $\left(1-\boldsymbol{H}(\lambda)_{i, i}\right)$ by their average $1-\operatorname{trace}(\boldsymbol{H}(\lambda)) / n$, the generalized cross-validation criterion is obtained.

$$
\begin{aligned}
\operatorname{GCV}(\lambda) & =n \sum_{i=1}^{n}\left(y_{i}-\widehat{m}\left(x_{i}\right)\right)^{2} /(n-\operatorname{trace}(\boldsymbol{H}(\lambda)))^{2} \\
& =n\|\boldsymbol{Y}-\boldsymbol{F} \hat{\boldsymbol{\beta}}\|^{2} /(n-\operatorname{trace}(\boldsymbol{H}(\lambda)))^{2}
\end{aligned}
$$

See Craven and Whaba (1979) and Ruppert et al. (2003), among others, for more details.

Using these criteria to select a value of $\lambda$ when the data may contain outliers is generally not recommended (see, for example, Cantoni and Ronchetti (2001) and references therein). Intuitively one can see that all observations $y_{i}, i=1, \ldots, n$ in $(17)$ are treated with equal importance. However, if, for some $1 \leq j \leq n$, the observation $y_{j}$ is atypical, we would not want to fit it well. In other words, regardless of the robustness of the estimator $\widehat{m}(x)$, the criteria above may select a value of $\lambda$ that results in an estimated $m\left(x_{j}\right)$ closer to $y_{j}$ than desired. For the case of M-type smoothing splines, using the concept of pseudo-data of Cox (1983), Cantoni and Ronchetti (2001) proposed to down-weight the terms in (16) according to their residuals. For S-penalized regression splines, Result 1 suggest that we can think of $\widehat{\boldsymbol{\beta}}_{S}$ as the solution to

$$
\min _{\boldsymbol{\beta}}\left\|\boldsymbol{W}\left(\widehat{\boldsymbol{\beta}}_{S}\right)^{1 / 2}(\boldsymbol{Y}-\boldsymbol{F} \boldsymbol{\beta})\right\|^{2}+\left(\lambda / \tau\left(\widehat{\boldsymbol{\beta}}_{S}\right)\right) \boldsymbol{\beta}^{t} \boldsymbol{D} \boldsymbol{\beta},
$$

where $\boldsymbol{W}\left(\widehat{\boldsymbol{\beta}}_{S}\right)$ and $\tau\left(\widehat{\boldsymbol{\beta}}_{S}\right)$ are given in Result 1 . The above representation suggests the use of the GCV criterion in (17) with response variable $\tilde{\boldsymbol{Y}}=\boldsymbol{W}\left(\widehat{\boldsymbol{\beta}}_{S}\right)^{1 / 2} \boldsymbol{Y}$, predictors $\tilde{\boldsymbol{F}}=\boldsymbol{W}\left(\widehat{\boldsymbol{\beta}}_{S}\right)^{1 / 2} \boldsymbol{F}$ and penalty term $\lambda / \tau\left(\widehat{\boldsymbol{\beta}}_{S}\right)$. Noting that some of the weights may be 
zero, we propose to select $\lambda$ by minimizing

$$
\operatorname{RGCV}(\lambda)=n_{w}\left\|\boldsymbol{W}(\widehat{\boldsymbol{\beta}})^{1 / 2}(\boldsymbol{Y}-\boldsymbol{F} \widehat{\boldsymbol{\beta}})\right\|^{2} /\left(n_{w}-\operatorname{trace}\left(\boldsymbol{H}_{\boldsymbol{S}}(\lambda)\right)\right)^{2},
$$

where

$$
\begin{aligned}
\boldsymbol{H}_{\boldsymbol{S}}(\lambda) & =\tilde{\boldsymbol{F}}\left(\tilde{\boldsymbol{F}}^{t} \tilde{\boldsymbol{F}}+\left(\lambda / \tau\left(\hat{\boldsymbol{\beta}}_{S}\right)\right) \boldsymbol{D}\right)^{-1} \tilde{\boldsymbol{F}}^{t} \\
& =\boldsymbol{W}\left(\widehat{\boldsymbol{\beta}}_{S}\right)^{1 / 2} \boldsymbol{F}\left(\boldsymbol{F}^{t} \boldsymbol{W}\left(\widehat{\boldsymbol{\beta}}_{S}\right) \boldsymbol{F}+\left(\lambda / \tau\left(\widehat{\boldsymbol{\beta}}_{S}\right)\right) \boldsymbol{D}\right)^{-1} \boldsymbol{F} \boldsymbol{W}\left(\widehat{\boldsymbol{\beta}}_{S}\right)^{1 / 2},
\end{aligned}
$$

and $n_{w}$ is the number of non-zero weights.

\section{$3 \quad$ Numerical results}

\subsection{Simulation settings}

The settings for the simulation study are as follows. The observations for the design variable $x_{1}, \ldots, x_{n}$ are generated from a uniform distribution on interval $[-1,1]$, for various sample sizes. These values are kept fixed for all settings to reduce simulation variability. The sample sizes taken are $n=25,100$ and 250 .

For the mean structure in (1) we have used the following functions, which represent a variety of shapes, $m_{1}(x)=\sin (\pi x), m_{2}(x)=\sin \left(2 \pi(1-x)^{2}\right), m_{3}(x)=x+x^{2}+x^{3}+x^{4}$, and $m_{4}(x)=-20+e^{3 x}$. Function $m_{2}$ is the same as used by Lee and Oh (2007) to facilitate a comparison with the results presented there.

For the error distribution we used five possibilities, ordered according to heaviness of their tails, (i) uniform distribution(-1,1), (ii) normal distribution $N\left(0,0.7^{2}\right)$, (iii) logistic distribution $(0,1)$, (iv) slash distribution, defined as $N(0,1) /$ uniform $(0,1)$, and (v) Cauchy distribution $(0,1)$. Both the Cauchy and slash distribution are heavy-tailed.

We compare three penalized estimation methods in this simulation study: (A) the nonrobust method for penalized regression spline estimation as in (5), using the method of penalized least squares (LS), (B) Penalized M-estimators as studied by Lee and Oh (2007). (C) the method proposed in this paper, using penalized S-estimators, and employing the algorithm as described in section 2.2. For the proposed method using penalized Sestimators we used the Tukey's biweight family of loss function $\rho_{d}(u)$ as in (13) with 
$d=1.547$. For the penalized M-estimators we used as suggested in Lee and Oh (2007), $\rho_{c}(t)$ as in $(7)$ with $c=1.345 \hat{\sigma}$, where $\hat{\sigma}$ is the median absolute deviation of residuals.

For all three methods, we used truncated cubic splines $(p=3)$ with $K=6,25$ or 35 knots (corresponding to sample sizes 25, 100 and 250), spread equally according to the quantiles of the data. The penalty parameter $\lambda$ is chosen by minimizing the generalized cross validation (GCV) criterion for the LS- and M-estimation methods. Robust generalized cross validation (RGCV) defined in section 2.3 is used for the S-estimation method.

For the proposed method of penalized S-estimation and the M-estimation method as proposed by Lee and Oh (2007) we set the tolerance level in the algorithm to $\epsilon=10^{-6}$. The maximum number of iterations was set to 500 .

To investigate the robustness of the methods against outliers, we randomly generated different percentages of outliers $(5 \%, 10 \%, 20 \%, 30 \%$ and $40 \%)$ for each of the simulated cases using either a normal distribution with mean 20 and standard deviation 20, to get scattered outliers, or with mean 20 and standard deviation 2 for a more concentrated cloud of outliers.

To give an impression on the variability of the obtained estimators, we plot in Figure 1, a scatter plot of one of the randomly generated data sets, together with the fitted values from the penalized LS-, M- and S-estimation methods. We used randomly generated data sets with mean function $m_{1}(x)$ and error distribution $N(0,1)$ for sample size $n=100$. Figure 1 (a) shows the situation without outliers, giving close correspondence between all three methods. In the situation of $30 \%$ of scattered outliers in Figure 1 (b), the drastic effects of the outliers are clearly visible for the penalized least squares method. A smaller effect is detected for the M-estimation method. In contrast to both penalized LS and M, the penalized S-estimator remains close to the true regression function, also in presence of outliers. 
(a)

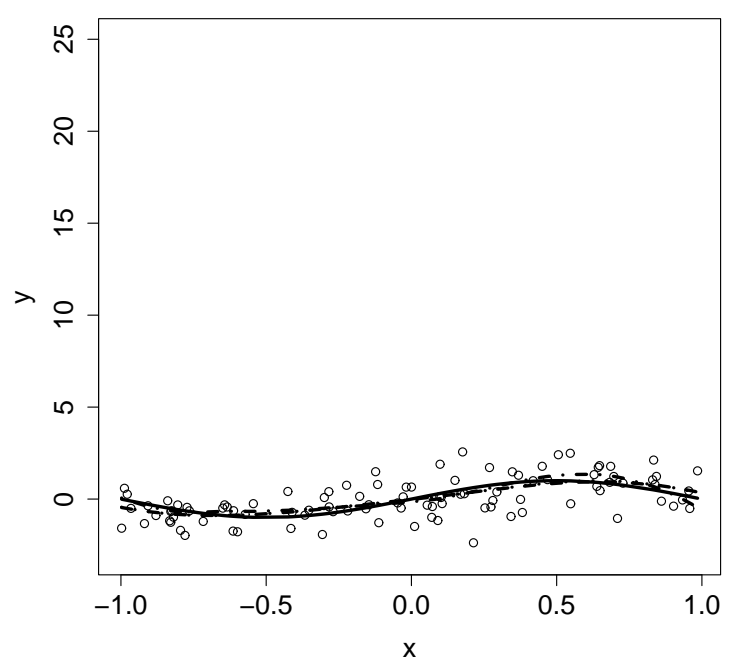

(b)

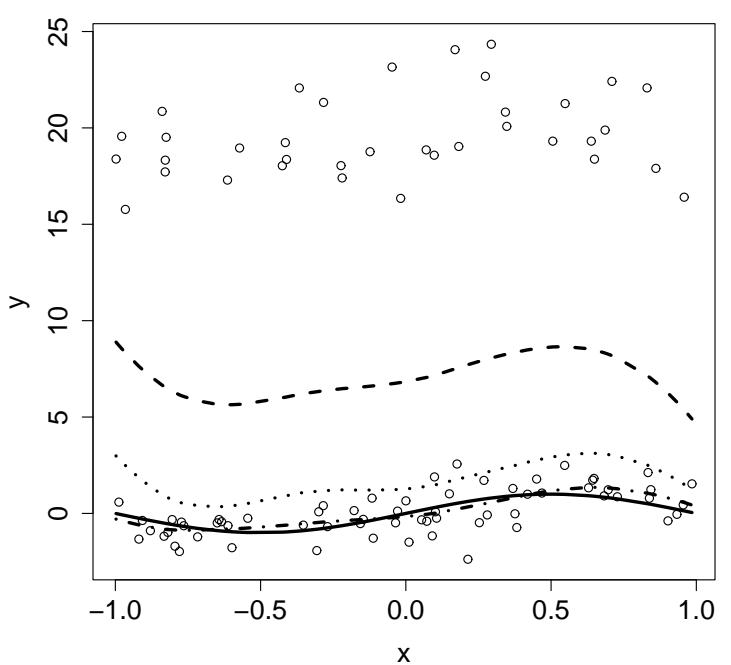

Figure 1: Fitted values (a) without outliers and (b) with $30 \%$ of outliers from $N\left(20,2^{2}\right)$. True function $\sin (\pi x)$ (solid line); fitted curves from penalized LS estimation (dashed); penalized M estimation (dotted) and penalized S estimation (dot-dashed).

\subsection{Simulation results}

The goodness of fit of the estimated model is quantified by computing the median average squared error and median absolute deviation of average squared error. Denoting $\widehat{m}_{j}\left(x_{i}\right)$ the estimated value of $m\left(x_{i}\right)$ for simulation $\operatorname{run} j(j=1, \ldots, J=1000)$, the average squared error (ASE) is defined by

$$
A S E_{j}=\frac{1}{n} \sum_{i=1}^{n}\left(m\left(x_{i}\right)-\widehat{m}_{j}\left(x_{i}\right)\right)^{2}, \quad j=1,2, \ldots, J .
$$

Table 1 presents summary values of the ASE (median and median absolute deviation) for the three estimation methods for the normal error distribution and with mean function $m_{1}$

In all cases, the median ASE of the proposed method of penalized S-estimation is smaller than that of the other two methods for samples with more than $10 \%$ of outliers. Note that Lee and Oh's (2007) method of penalized M-estimation works better for samples with $5 \%$ and $10 \%$ of outliers. 
For the penalized least squares and penalized M-estimators, the ASE is clearly increasing with the percentage of outliers increasing. For penalized S-estimation, the ASE values tend to be quite stable, only increasing near a high fraction of outliers $(>40 \%)$. As expected, the goodness of fit as measured by the ASE values improves for larger sample sizes.

Table 1 clearly shows that the penalized least squares method may already break down with only $5 \%$ of outliers. For the proposed method of penalized S-estimation, the simulated ASE values are relatively small even with $40 \%$ of scattered outliers for sample sizes $n=100$ and $n=250$. For $n=25$ a clearer increase (breakdown) is observed for the penalized S-estimation method when the presence of outliers reaches $40 \%$ of the sample size. For penalized M-estimation, the breakdown arrives earlier, showing the need for taking the scale into consideration in the fitting method and working with a bounded $\rho$-function.

Table 1: Median and Median absolute deviation (between parenthesis) of the average squared error ASE for penalized least squares (LS), penalized M (M) and penalized S (S) estimation for data generated with mean structure $m_{1}(x)$, error terms from a $N\left(0,0.7^{2}\right)$ distribution, and for different sample sizes. We consider different percentages $\varepsilon$ of outliers generated from $N\left(20,2^{2}\right)$.

\begin{tabular}{lccccccccc}
\hline$\varepsilon$ & & $n=25$ & \multicolumn{3}{c}{$n=100$} & \multicolumn{3}{c}{$n=250$} \\
& $L S$ & $M$ & $S$ & $L S$ & $M$ & $S$ & $L S$ & $M$ & $S$ \\
\hline $0 \%$ & 0.07 & 0.08 & 0.18 & 0.02 & 0.02 & 0.07 & 0.01 & 0.01 & 0.04 \\
& $(0.05)$ & $(0.05)$ & $(0.13)$ & $(0.01)$ & $(0.01)$ & $(0.05)$ & $(0.01)$ & $(0.01)$ & $(0.02)$ \\
$5 \%$ & 2.31 & 0.10 & 0.21 & 1.57 & 0.03 & 0.08 & 1.35 & 0.02 & 0.04 \\
& $(3.25)$ & $(0.08)$ & $(0.17)$ & $(1.19)$ & $(0.02)$ & $(0.05)$ & $(0.69)$ & $(0.01)$ & $(0.02)$ \\
$10 \%$ & 7.48 & 0.13 & 0.21 & 5.12 & 0.06 & 0.07 & 4.56 & 0.04 & 0.03 \\
& $(7.07)$ & $(0.12)$ & $(0.17)$ & $(2.84)$ & $(0.04)$ & $(0.05)$ & $(1.73)$ & $(0.02)$ & $(0.02)$ \\
$20 \%$ & 22.97 & 0.46 & 0.24 & 18.45 & 0.21 & 0.06 & 16.91 & 0.16 & 0.03 \\
& $(16.40)$ & $(0.55)$ & $(0.22)$ & $(7.01)$ & $(0.15)$ & $(0.04)$ & $(4.18)$ & $(0.07)$ & $(0.02)$ \\
$30 \%$ & 45.00 & 17.19 & 0.35 & 38.77 & 1.04 & 0.05 & 37.32 & 0.77 & 0.02 \\
& $(24.50)$ & $(25.03)$ & $(0.42)$ & $(12.00)$ & $(0.97)$ & $(0.03)$ & $(7.06)$ & $(0.44)$ & $(0.01)$ \\
$40 \%$ & 75.64 & 68.55 & 32.47 & 66.78 & 42.06 & 0.07 & 66.02 & 12.83 & 0.02 \\
& $(34.65)$ & $(54.86)$ & $(48.03)$ & $(16.75)$ & $(49.37)$ & $(0.06)$ & $(9.74)$ & $(15.44)$ & $(0.02)$ \\
\hline
\end{tabular}

To give an impression on the variability of the obtained estimators, we plot the box 
plots of log scale of ASEs of the simulation samples from penalized least squares, penalized $\mathrm{M}$ and penalized S estimation in Figures 2 and 3 for the data with outliers $N\left(20,2^{2}\right)$ and $N\left(20,20^{2}\right)$ respectively. These plots show that the ASEs of the penalized S-estimator remain stable as the proportion of contamination increases. Even though they become more variable for $40 \%$ of outliers, the median is still at the same level as before. The penalized LS-estimator's ASEs grow very rapidly. Similarly, the penalized M-estimator's ASEs grow rapidly after $10 \%$ of outliers. These results are confirming that the penalized M-estimation method works better with less than $10 \%$ of outliers, while the penalized S-estimation method works well for all considered percentages of outliers.

(a)

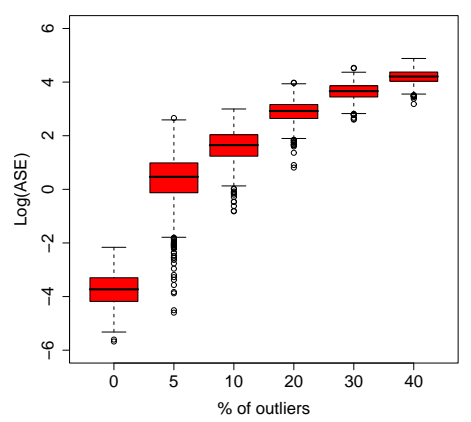

(b)

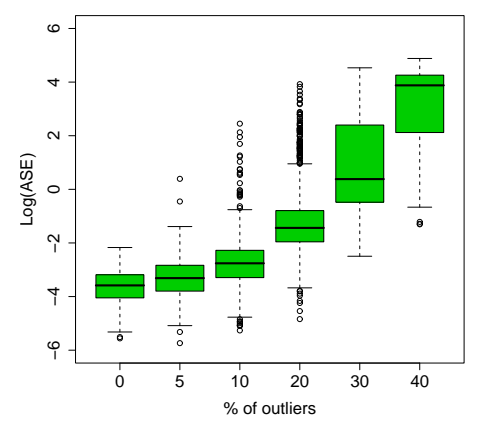

(c)

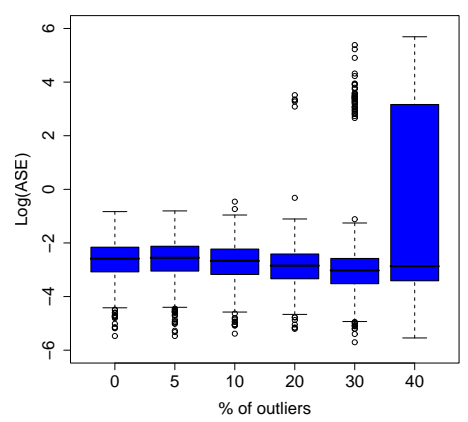

Figure 2: Box plots of ASEs using (a) penalized LS-estimation, (b) penalized M-estimation and (c) penalized S-estimation for samples with mean structure $m_{1}(x)$, error distribution $N\left(0,0.7^{2}\right)$ and outliers $N\left(20,2^{2}\right)$, and sample size $n=100$.

(a)

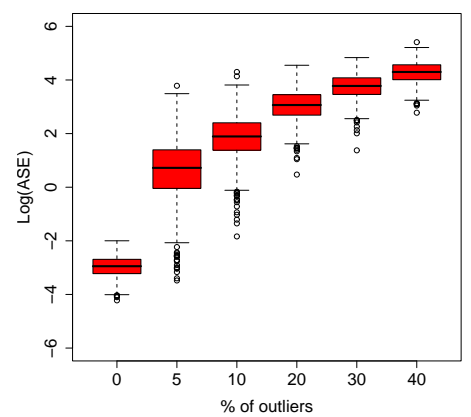

(b)

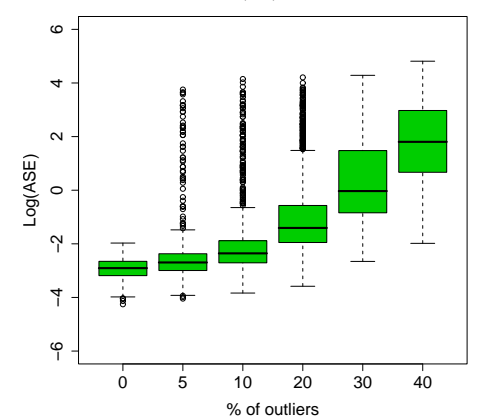

(c)

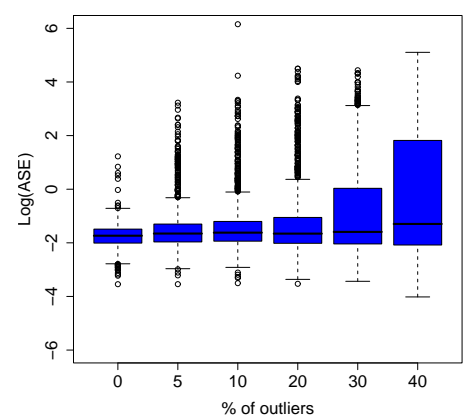

Figure 3: Box plots of ASEs using (a) penalized LS-estimation, (b) penalized M-estimation and (c) penalized S-estimation for samples with mean structure $m_{1}(x)$, error distribution $N\left(0,0.7^{2}\right)$ and scattered outliers $N\left(20,20^{2}\right)$, and sample size $n=100$. 
Next we compare the effects of the different error distributions on the performance of the estimates. The results are shown in Table 2 for sample size $n=100$ and true mean function $m_{1}$. The proposed method gives the smallest median ASE values for all considered error distributions if there are more than $20 \%$ outliers. Penalized M-estimation works better for the samples with $5 \%$ and $10 \%$ of outliers for uniform and logistic error distributions. For LS-estimation and M-estimation methods, the ASE values are relatively large for heavy-tailed distributions (Slash and Cauchy). Note that in absence of outliers $(\varepsilon=0 \%)$ the method of penalized S-estimation works better than LS at heavy tailed distributions.

Table 2: Median and median absolute deviation (between parenthesis) of the average squared error ASE for penalized least squares (LS), penalized M (M) and penalized S (S) estimation for data generated with mean structure $m_{1}(x)$, error terms from different distributions, and for sample sizes $n=100$ with different percentages $\varepsilon$ of outliers generated from $N\left(20,2^{2}\right)$.

\begin{tabular}{lccccccccccccc}
\hline$\varepsilon$ & \multicolumn{3}{c}{ Uniform } & \multicolumn{3}{c}{ Logistic } & \multicolumn{3}{c}{ Slash } & \multicolumn{3}{c}{ Cauchy } \\
& $L S$ & $M$ & $S$ & $L S$ & $M$ & $S$ & $L S$ & $M$ & $S$ & $L S$ & $M$ & $S$ \\
\hline $0 \%$ & 0.02 & 0.02 & 0.15 & 0.14 & 0.14 & 0.34 & 6.69 & 0.30 & 0.32 & 4.92 & 0.16 & 0.12 \\
& $(0.0)$ & $(0.0)$ & $(0.1)$ & $(0.1)$ & $(0.1)$ & $(0.2)$ & $(8.4)$ & $(0.2)$ & $(0.2)$ & $(6.1)$ & $(0.1)$ & $(0.1)$ \\
$5 \%$ & 1.62 & 0.03 & 0.15 & 1.75 & 0.19 & 0.33 & 8.95 & 0.47 & 0.32 & 6.71 & 0.24 & 0.12 \\
& $(1.2)$ & $(0.0)$ & $(0.1)$ & $(1.3)$ & $(0.1)$ & $(0.2)$ & $(9.8)$ & $(0.4)$ & $(0.2)$ & $(7.2)$ & $(0.2)$ & $(0.1)$ \\
$10 \%$ & 5.27 & 0.04 & 0.13 & 5.44 & 0.31 & 0.31 & 12.8 & 0.81 & 0.31 & 10.3 & 0.42 & 0.12 \\
& $(2.9)$ & $(0.0)$ & $(0.1)$ & $(3.0)$ & $(0.2)$ & $(0.2)$ & $(11)$ & $(0.7)$ & $(0.2)$ & $(8.8)$ & $(0.3)$ & $(0.1)$ \\
$20 \%$ & 18.4 & 0.16 & 0.10 & 18.5 & 1.20 & 0.28 & 25.5 & 3.52 & 0.31 & 23.5 & 2.07 & 0.14 \\
& $(7.0)$ & $(0.1)$ & $(0.1)$ & $(7.0)$ & $(0.9)$ & $(0.2)$ & $(16)$ & $(3.0)$ & $(0.2)$ & $(13)$ & $(1.8)$ & $(0.1)$ \\
$30 \%$ & 39.4 & 0.87 & 0.06 & 39.5 & 6.78 & 0.26 & 46.1 & 23.8 & 0.42 & 45.1 & 16.4 & 0.21 \\
& $(11)$ & $(0.8)$ & $(0.0)$ & $(11)$ & $(6.3)$ & $(0.2)$ & $(22)$ & $(23)$ & $(0.4)$ & $(19)$ & $(18)$ & $(0.2)$ \\
$40 \%$ & 68.3 & 46.8 & 0.07 & 68.6 & 58.2 & 0.49 & 73.4 & 71.1 & 56.5 & 73.3 & 69.3 & 33.3 \\
& $(15)$ & $(48)$ & $(0.1)$ & $(16)$ & $(32)$ & $(0.6)$ & $(27)$ & $(21)$ & $(83)$ & $(25)$ & $(21)$ & $(49)$ \\
\hline
\end{tabular}

We have further checked our proposed method with that of Lee and Oh (2007) using the same regression function $m_{2}$ as in their paper. We generated errors $\varepsilon_{i}$ from a normal distribution, and included different percentages of outliers for sample size $n=100$. For each of these settings we computed the ASE over 1000 simulation runs; the results are presented in Table 3. All previous findings are confirmed. The S-estimation method 
does a better job than M-estimation when there are more than $20 \%$ of outliers. The M-estimation method works better for the cases with $5 \%$ and $10 \%$ of outliers. This holds for the goniometric $\left(m_{2}\right)$, the polynomial $\left(m_{3}\right)$, and the exponential $\left(m_{4}\right)$ mean functions.

Table 3: Median and median absolute deviation (between parenthesis) of the average squared error ASE for penalized least squares (LS), penalized M (M) and penalized S (S) estimation for data generated from functions $m_{2}, m_{3}$ and $m_{4}$ with error terms from $N\left(0,0.7^{2}\right)$ for sample size $n=100$ with different percentages $\varepsilon$ of outliers generated from $N\left(20,2^{2}\right)$.

\begin{tabular}{lccccccccc}
\hline$\varepsilon$ & & $m_{2}$ & & \multicolumn{3}{c}{$m_{3}$} & & & $m_{4}$ \\
& $L S$ & $M$ & $S$ & $L S$ & $M$ & $S$ & $L S$ & $M$ & $S$ \\
\hline $0 \%$ & 0.25 & 0.27 & 0.38 & 0.02 & 0.02 & 0.07 & 0.04 & 0.04 & 0.14 \\
& $(0.02)$ & $(0.02)$ & $(0.11)$ & $(0.01)$ & $(0.01)$ & $(0.05)$ & $(0.02)$ & $(0.02)$ & $(0.07)$ \\
$5 \%$ & 1.87 & 0.28 & 0.40 & 1.47 & 0.03 & 0.08 & 5.30 & 0.05 & 0.13 \\
& $(1.17)$ & $(0.03)$ & $(0.13)$ & $(1.09)$ & $(0.02)$ & $(0.05)$ & $(4.00)$ & $(0.02)$ & $(0.07)$ \\
$10 \%$ & 5.44 & 0.32 & 0.37 & 4.83 & 0.05 & 0.07 & 17.3 & 0.07 & 0.12 \\
& $(2.82)$ & $(0.06)$ & $(0.13)$ & $(2.73)$ & $(0.04)$ & $(0.05)$ & $(9.88)$ & $(0.04)$ & $(0.07)$ \\
$20 \%$ & 18.6 & 0.61 & 0.33 & 17.4 & 0.20 & 0.06 & 63.0 & 0.24 & 0.11 \\
& $(7.08)$ & $(0.27)$ & $(0.12)$ & $(6.67)$ & $(0.14)$ & $(0.04)$ & $(24.2)$ & $(0.16)$ & $(0.06)$ \\
$30 \%$ & 38.5 & 2.11 & 0.31 & 36.9 & 1.05 & 0.05 & 133 & 1.19 & 0.11 \\
& $(11.8)$ & $(1.75)$ & $(0.12)$ & $(11.2)$ & $(0.97)$ & $(0.04)$ & $(38.0)$ & $(1.10)$ & $(0.06)$ \\
$40 \%$ & 66.4 & 45.6 & 0.38 & 63.5 & 38.8 & 0.06 & 229 & 112 & 0.11 \\
& $(16.2)$ & $(44.9)$ & $(0.25)$ & $(15.4)$ & $(46.4)$ & $(0.06)$ & $(54.6)$ & $(161)$ & $(0.08)$ \\
\hline
\end{tabular}

\section{Balloon data}

In this section, we have used the balloon data set from the R software's library ftnonpar. The data are radiation measurements from the sun, taken from a flight of a weather balloon. Due to the rotation of the balloon, or for some other reasons, outliers were introduced because the measuring device was occasionally blocked from the sun. The response variable $Y$ is a radiation measurement and the explanatory variable $x$ is the index of the measurement. The sample size equals 4,984. We took $K=35$ knots spread equally, and scaled the value $\lambda$ according to the GCV and RGCV methods in section 2.3. We obtained $\lambda=0.04$ for penalized LS-estimation method and $\lambda=0.1$ for penalized Mand S-estimation method. 


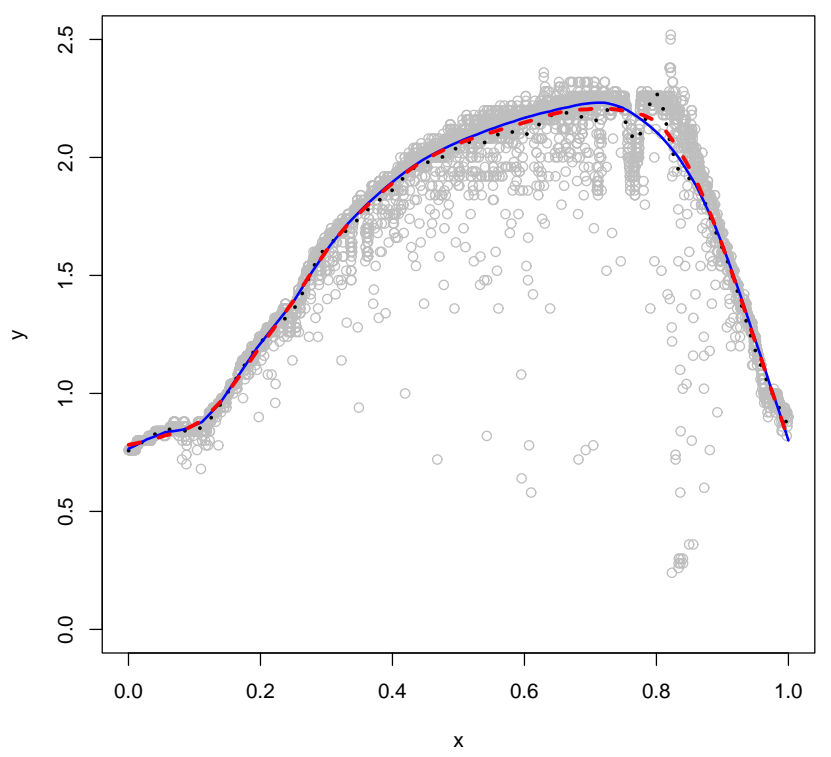

Figure 4: Fitted values for the balloon data. Penalized LS method (dotted), penalized S (solid) and penalized M (dashed).

Displayed in Figure 4 are regression estimates obtained by the penalized LS method, our proposed method of penalized S-estimation and penalized M-estimation. The nonrobust curve suffers from the presence of the outliers, which is clearly visible around the value $x=0.8$. That is, the estimated curve was pulled upwards, in the direction of the outliers. The robust methods do not suffer from this phenomenon.

\section{Conclusions}

In this paper a simple and effective method is proposed for robust fitting penalized regression spline models. Generally, smoothing methods may be influenced by outliers. The proposed method is easy to implement and fast to converge. Penalized S-estimators improve on penalized least squares regression splines and penalized M-estimators. The procedure performs very well in all of our numerical examples. The penalized M-estimation works better for the cases with a small percentage of contamination but penalized S-estimation works well for higher percentage of contamination too. 


\section{References}

Beaton, A. and Tukey, J. (1974). The fitting of power series, meaning polynomials, illustrated on band-spectroscopic data. Technometrics, 16:147-185.

Besse, P., Cardot, H., and Ferraty, F. (1997). Simultaneous nonparametric regression of unbalanced longitudial data. Comp. Statist. Data Anal., 24:255-270.

Cantoni, E. and Ronchetti, E. (2001). Resistant selection of the smoothing parameter for smoothing splines. Stat. Comput., 11(2):141-146.

Claeskens, G., Krivobokova, T., and Opsomer, J. (2007). Asymptotic properties of penalized spline estimators. Research Report KBI, ORSTAT, K.U.Leuven.

Cox, D. D. (1983). Asymptotics for M-type smoothing splines. Ann. Statist., 11(2):530551.

Craven, P. and Whaba, G. (1979). Smoothing noisy data with spline functions. $\mathrm{Nu}$ merische Mathematik, 31:377-403.

Davies, L. (1990). The asymptotics of S-estimators in the linear regression model. Ann. Stat., 18(4):1651-1675.

Donoho, D. and Huber, P. (1983). The notion of breakdown-point. In Bickel, P., Doksum, K., and Hodges, J. J., editors, A Festschrift for Erich L. Lehmann. Wadsworth.

Eilers, P. H. C. and Marx, B. D. (1996). Flexible smoothing with $B$-splines and penalties. Stat. Science, 11(2):89-121. With comments and a rejoinder by the authors.

Hall, P. and Jones, M. C. (1990). Adaptive M-estimation in nonparametric regression. Ann. Statist., 18:1712-1728.

Hall, P. and Opsomer, J. (2005). Theory for penalized spline regression. Biometrika, 92:105-118.

Härdle, W. and Gasser, T. (1984). Robust non-parametric function fitting. J. R. Statist. Soc. B, 46:42-51.

Huber, P. (1964). Robust estimation of a location parameter. Ann. Math. Stats., 35:73101.

Huber, P. (1979). Robust smoothing. In Wilkinson, G. and Launer, R., editors, Robustness in Statistics. Academic Press.

Kauermann, G., Krivobokova, T., and Fahrmeir, L. (2007). Some asymptotic results on generalized penalized spline smoothing. Research Report KBI-0733, ORSTAT, K.U.Leuven.

Kelly, C. and Rice, J. (1990). Monotone smoothing with application to dose-response curves and the assessment of synergism. Biometrics, 46:1071-1085. 
Lee, T. C. M. and Oh, H.-S. (2007). Robust penalized regression spline fitting with application to additive mixed modeling. Comput. Statist., 22(1):159-171.

Maronna, R. A., M. R. D. and Yohai, V. J. (2006). Robust Statistics Theory and Methods. John Wiley and Sons,Ltd.

Maronna, R. A. and Yohai, V. J. (1991). The breakdown point of simultaneous general M-estimates of regression and scale. Journal of the American Statistical Association, 86(415):699-703.

Oh, H.-S., Nychka, D. W., Brown, T., and Charbonneau, P. (2004). Period analysis of variable stars by robust smoothing. Appl. Statist., 53(1):15-30.

Oh, H.-S., Nychka, D. W., and Lee, T. C. M. (2007). The role of pseudo data for robust smoothing with application to wavelet regression. Biometrika, 94(4):893-904.

O'Sullivan, F. (1986). A statistical perspective on ill-posed inverse problems. Stat. Science, 1:505-527. With discussion.

R Development Core Team (2008). R: A Language and Environment for Statistical Computing. R Foundation for Statistical Computing, Vienna, Austria. ISBN 3-900051-07-0.

Rousseeuw, P. and Yohai, V. (1984). Robust regression by means of S-estimators. In Robust and nonlinear time series analysis (Heidelberg, 1983), volume 26 of Lecture Notes in Statist., pages 256-272. Springer, New York.

Ruppert, D., Wand, M., and Carroll, R. (2003). Semiparametric Regression. Cambridge University Press, Cambridge, UK.

Salibian-Barrera, M. and Yohai, V. (2006). A fast algorithm for S-regression estimates. Journal of Computational and Graphical Statistics, 15:414-427.

Silverman, B. W. (1985). Some aspects of the spline smoothing approach to nonparametric regression curve fitting. J. R. Statist. Soc. B, 47(1):1-52. 